\title{
SINGLE-NANOWIRE SI SOLAR CELLS
}

\author{
M. D. Kelzenberg, D. B. Turner-Evans, B. M. Kayes, M. A. Filler, M. C. Putnam, N. S. Lewis, H. A. Atwater \\ California Institute of Technology, Pasadena, CA 91125
}

\begin{abstract}
Solar cells based on arrays of CVD-grown Si nano- or micro-wires are being considered as a potentially low-cost route to implementing a vertical multijunction cell design via radial $p-n$ junctions. This geometry has been predicted to enable efficiencies competitive with planar multicrystalline Si designs, while reducing the materials and processing costs of solar cell fabrication [1]. To further assess the potential efficiency of cells based on this design, we present here experimental measurements of minority carrier diffusion lengths and surface recombination rates within nanowires via fabrication and characterization of single-wire solar cell devices. Furthermore, we consider a potential Si wire array-based solar cell design, and present device physics modeling of single-wire photovoltaic efficiency. Based on experimentally observed diffusion lengths within our wires, we model a radial junction wire solar cell capable of $17 \%$ photovoltaic energy conversion efficiency.
\end{abstract}

\section{INTRODUCTION}

Crystalline silicon is by far the dominant material used for worldwide photovoltaic energy conversion and solar cell manufacture. However, commercial cells are already approaching the practical limit of energy conversion efficiency [2]. Thus, in order to reduce the overall cost per watt of solar cell production, many efforts are being directed at reducing the costs associated with manufacturing efficient crystalline Si cells.

Solar cells based on arrays of vertical Si micro- or nanowires have been proposed as one possible route to lower production costs, while still obtaining efficiencies competitive with planar multicrystalline Si cells. The reason for this is twofold. Firstly, materials costs can be reduced because the radial junction geometry tolerates the use of lower-purity silicon with minimal efficiency loss compared to a planar geometry [1]. Secondly, processing costs could be reduced by employing thin-film techniques such as chemical vapor deposition (CVD) of the active layer and roll-to-roll sheet processing. Nanowire films may be grown from chlorosilane feedstock by the vaporliquid-solid (VLS) [3] CVD technique, and may be grown on, or transferred to [4], inexpensive flexible substrates.

Within each Si wire of the proposed vertical wire array solar cell there is a radial $p-n$ (or $n-p)$ junction. This decouples the vertical process of light absorption from the radial process of charge collection, much like the vertical multijunction (VMJ) geometry [5]. This allows for near- unity internal quantum efficiency across the entire abovebandgap solar spectrum. The wires can easily be made long enough to absorb all above-bandgap illumination, while remaining small enough in diameter to efficiently collect all photogenerated minority carriers. Despite optimal charge collection, however, this design cannot accommodate arbitrarily low diffusion lengths. As the wire radius decreases, the junction area increases and the cell becomes increasingly sensitive to shunting, which also increases with lower diffusion lengths. The drop in cell operating voltage may decrease overall cell performance despite enhanced current collection. Thus, the design of an efficient Si wire solar cell requires careful optimization of wire radius in addition to the parameters which must be optimized on a planar junction cell. In this work we expand on prior analytical modeling [1] by considering 2dimensional carrier transport within a single-wire Si solar cell model based on a wire array design which might realistically be fabricated.

The critical parameter for maximizing the efficiency of the single-wire radial junction solar cell is the minority carrier diffusion length. Hole diffusion lengths of up to $80 \mathrm{~nm}$, limited by surface recombination, have been inferred from electron beam-induced current measurements on $100 \mathrm{~nm}$ diameter, Au-catalyzed Si nanowires [6]. We have reported diffusion lengths of up to $4 \mu \mathrm{m}$ within $\sim 1 \mu \mathrm{m}$ diameter, Au-catalyzed, single-wire Si solar cells with axial rectifying junctions [7]. Radial $p$-i-n junction singlenanowire solar cells [8] have been reported with device efficiencies up to $3.4 \%$. However, the single-wire cells in [7] and [8] were not subject to vertical illumination because the wires had been removed from the growth substrate and were lying horizontally. We now turn our attention to estimating the efficiency limits of the radial p-n junction Si wire solar cell, starting with continued characterization of diffusion lengths, lifetimes, and surface recombination rates.

\section{EXPERIMENTAL}

Micro- and nanowires for photovoltaic applications [9] were grown by the VLS process using $\mathrm{Au}$ and $\mathrm{Ni}$ catalyst metals. TEM images obtained for similar Au-catalyzed wires from our reactor indicated that they are singlecrystalline, and grow vertically in the $<111\rangle$ direction. Wires were removed from the growth substrate by sonication in isoproponal, and then deposited (horizontally) on an insulating substrate $\left(\mathrm{n}^{+} \mathrm{Si}\right.$ wafer with $100 \mathrm{~nm} \mathrm{Si}{ }_{3} \mathrm{~N}_{4}$.) Four-probe electrical contacts to single wires were formed using aligned photolithography, metallization, and liftoff. Al was evaporated as the contact metal, followed by a 

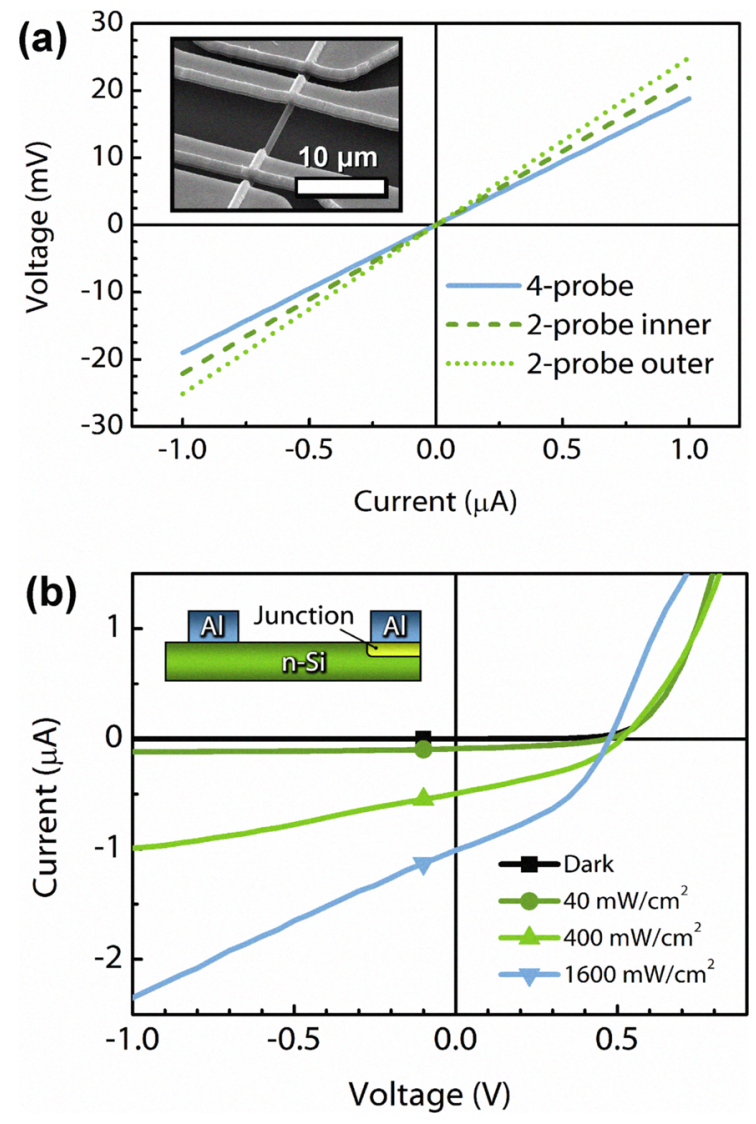

Fig. 1. (a) Typical I-V measurement of electrically contacted single-wire device, with inset SEM image of the device. (b) I-V measurement of single-wire device which has had one contact converted to a rectifying junction, showing photovoltaic behavior under varying laser illumination at $514 \mathrm{~nm}$.

layer of $\mathrm{Ag}$ to supplement the total metal layer thickness. The contacted wires ranged in diameter from $200 \mathrm{~nm}$ to $1.2 \mu \mathrm{m}$.

The electrical properties of each wire and contact were characterized by 2-, 3-, and 4-probe I-V measurements, as shown in figure 1(a). The as-deposited Al contacts were generally ohmic, with contact resistance less than wire resistance. Effective bulk resistivities were calculated for each wire device based on resistance and the dimensions of the wire observed in SEM imaging, assuming uniform conductivity. Effective doping levels were then inferred based on bulk mobility values for crystalline Si. For Au-catalyzed wires, electrically active doping levels ranged from $10^{17}$ to $10^{18} \mathrm{~cm}^{-3}$, while Ni-catalyzed wires varied from $10^{15}$ to $10^{16} \mathrm{~cm}^{-3}$. To determine carrier type, the underlying Si substrate was used as a gate to induce depletion and accumulation within the wire devices. Both sets of wires exhibited $n$-type behavior.
Single-wire photovoltaic devices were created by inducing rectifying junctions beneath one of the Al contacts to the Au-catalyzed wires. A voltage was applied between two adjacent outer contacts, allowing current to flow through the enclosed wire segment. The voltage was slowly increased until the wire segment was destroyed, as shown in figure 2(b). In successful cases, the inner contact to the remaining portion of the wire exhibited rectifying behavior, with ideality factors ranging from $2-3.5$. The rectification is likely due to the formation of a Schottky barrier at the contact, the formation of an Al/Si p-n alloy junction, or a combination thereof. These devices exhibited photovoltaic behavior, with photovoltages up to $0.5 \mathrm{~V}$ under laser illumination. The highest solar energy conversion efficiency observed was $0.49 \%$ under simulated AM 1.5G illumination. Open circuit voltages of up to 190 $\mathrm{mV}$ and short circuit current densities of up to $7.8 \mathrm{~mA} / \mathrm{cm}^{2}$ were observed.

Scanning photocurrent microscopy (SPCM, or light beam induced current, LBIC) experiments were performed on the single-wire solar cell devices to study minority carrier diffusion and recombination processes. A $650 \mathrm{~nm}$ laser source was focused to a $1 \mu \mathrm{m}$ spot through the objective of a confocal microscope (figure $2 \mathrm{a}$ ), and photocurrent was recorded as a function of illumination position at various applied biases, shown in figures 2 (c) and (d). Due to the rectifying junction at one end of the wire device, the minority carrier diffusion length could be directly observed as the exponential decay rate of photocurrent as the injection position was swept towards the opposite (ohmic) contact. For the Au-catalyzed wires, these decay lengths did not vary with applied bias, confirming minoritycarrier diffusion-limited transport within the wires. Diffusion lengths in Au-catalyzed wires with diameters near 1 $\mu \mathrm{m}$ have been observed ranging from $2-4 \mu \mathrm{m}$. The rectifying junction formation technique was not successful in wires with diameters less than $800 \mathrm{~nm}$.

For each observed diffusion length, the effective minority (hole) carrier lifetimes were calculated based on doping-dependent mobility values for crystalline Si. Lifetimes ranged from 6 to $25 \mathrm{~ns}$, which are comparable to expected lifetimes for $\mathrm{Si}$ doped with $\mathrm{Au}$ at its solid solubility limit at wire growth temperatures. [10] By assuming, instead, that recombination occurs only at the surface of the wire, one can calculate an upper limit on effective surface recombination velocity [11]. This calculation yields effective surface recombination velocities of $800-2000$ $\mathrm{cm} \mathrm{s}^{-1}$.

The SPCM technique has also been applied to Nicatalyzed wires, indicating generally longer diffusion lengths than for Au-catalyzed wires. This is expected, since $\mathrm{Au}$ forms a deep level trap within $\mathrm{Si}$ [10] while $\mathrm{Ni}$ remains relatively inert as long as it does not form a precipitate [12]. This is encouraging from a photovoltaics perspective, given the difference between the abundance and costs of Au vs. Ni. 

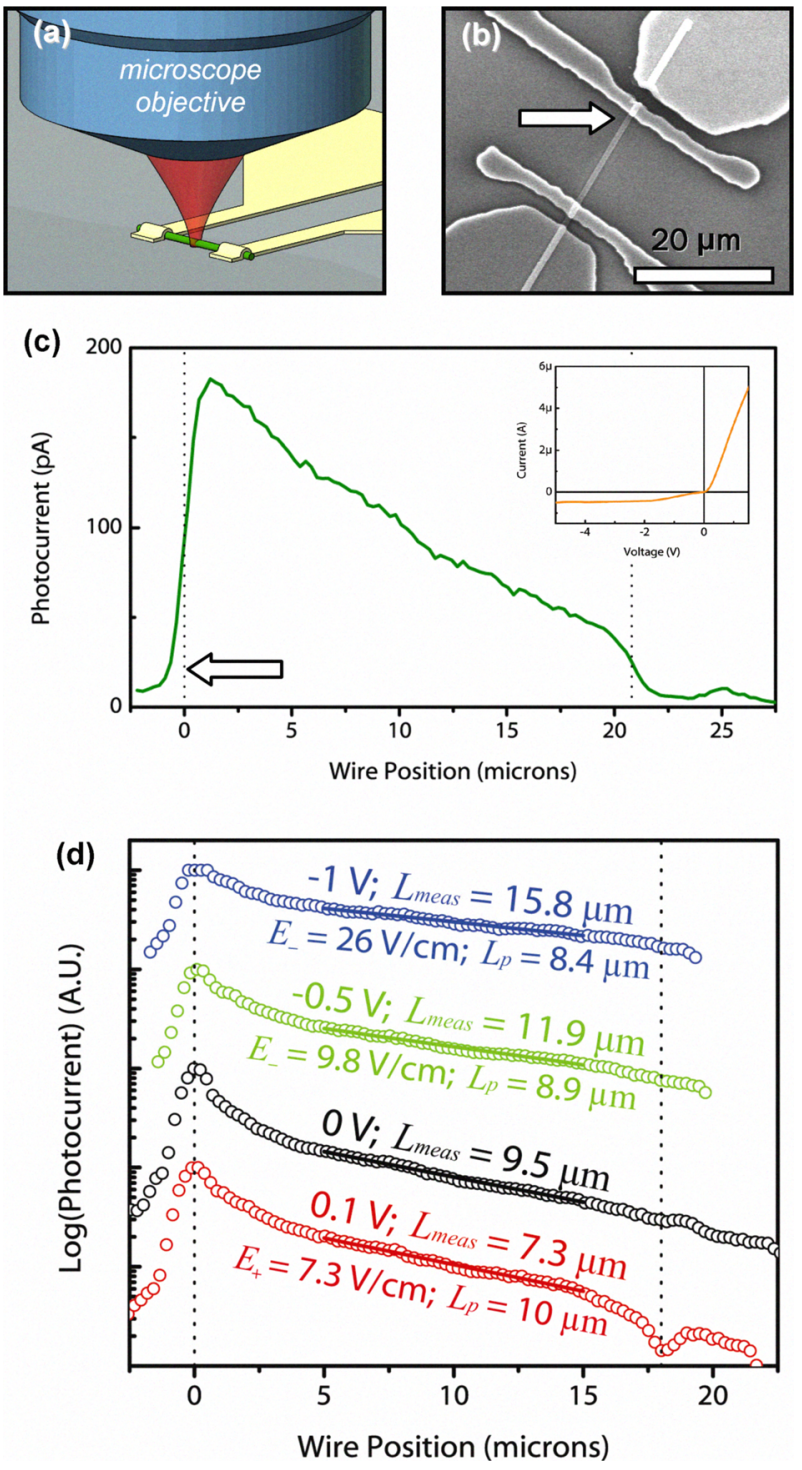

Fig. 2. (a) Simplified schematic of the SPCM/LBIC experiment, illustrating confocal illumination. (b) SEM image of a Ni-catalyzed single-wire photovoltaic device, with arrow indicating the location of the rectifying contact. (c) SPCM linescan profile of short-diode device obtained at $0 \mathrm{~V}$ bias, showing approximately linear decay of photocurrent away from the junction. The arrow indicates the junction location. Inset: I-V of typical Ni-catalyzed Si wire device. (d) Log-scale SPCM linescans of long-diode device obtained at various biases, showing inferred hole diffusion lengths.

However, experimental difficulties prevented as conclusive a study of diffusion length within Ni-catalyzed wires as was performed on the Au-catalyzed wires. This was due in part to the generally lower observed doping levels of the Ni-catalyzed wires, which made repeatable formation of ohmic and rectifying contacts difficult. At these levels, the junction formation procedure was rarely successful in creating rectifying junctions, appearing instead to only slightly modify the barrier height or interface charge density at the original contact. Furthermore at these doping levels, the band bending due to contacts, depletion regions, and surface charge extends further into the wire device, potentially complicating the assumption of quasi-neutrality within the wire.

Some Ni-catalyzed devices were successfully created which exhibited suitable rectifying behavior at one contact, and suitable ohmic behavior at the other. The devices which had $10 \mu \mathrm{m}$ electrode spacing generally exhibited linear, rather than exponential, spatial decay profiles under SPCM. This is characteristic of a short diode, in which the diffusion length exceeds the device length. In an attempt to extract the diffusion length, contact spacing was increased to $20 \mu \mathrm{m}$. Some devices exhibited exponential SPCM profiles (shown in figure 2d) yet other devices exhibited linear or sub-linear SPCM profiles indicative of diffusion lengths near or greater than $20 \mu \mathrm{m}$ (figure 2c.) An exemplary Ni-catalyzed long diode device is shown in figure $2 \mathrm{~d}$. Here, the observed diffusion length at non-zero biases have been corrected to account for electric fieldenhanced diffusion[13], due to the high resistance experienced by majority carrier currents at these doping levels. The electric field is calculated based on the dark I-V properties of the device and inferred resistivity. The correction yields a reasonably consistent diffusion length of approximately $10 \mu \mathrm{m}$. In directly comparing this result to that for the more heavily doped, Au-catalyzed wires, it is important to consider the difference in mobility between these wire batches. For $\mathrm{n}$-type $\mathrm{Si}$, a given minority carrier lifetime yields approximately double the diffusion length at $10^{16}$ $\mathrm{cm}^{-3}$ doping than at $10^{18} \mathrm{~cm}^{-3}[14]$.

Further experimental work is required to fully characterize diffusion length, carrier lifetimes, and surface recombination within $\mathrm{Ni}$ - and Au-catalyzed wires. However, we have observed diffusion lengths of up to $4 \mu \mathrm{m}$ in $\mathrm{Au}$ catalyzed Si wires. And despite the difficulties encountered in measuring diffusion lengths within Ni-catalyzed wires, we have seen strong experimental evidence that diffusion lengths are at least $10 \mu \mathrm{m}$, the longest reported diffusion length in VLS-grown Si wires to date. We have also inferred upper limits on wire surface recombination rates. These observations enable a more thorough investigation of Si wire-based photovoltaic performance, based on experimentally measured properties and physically achievable cell geometries.

\section{DEVICE PHYSICS MODELING}

To more accurately predict and optimize the performance potential of Si wire-based cells, we have employed the Synopsis TCAD software suite, including Sentaurus Device simulator (Version A-2007.12), to develop a device physics model of single-wire Si solar cell. Figure 3(a) illustrates a proposed $\mathrm{Si}$ wire array cell design featuring radial $p-n$ junctions, and figure $3(b)$ illustrates the singlewire equivalent cell studied here. The model is specified 

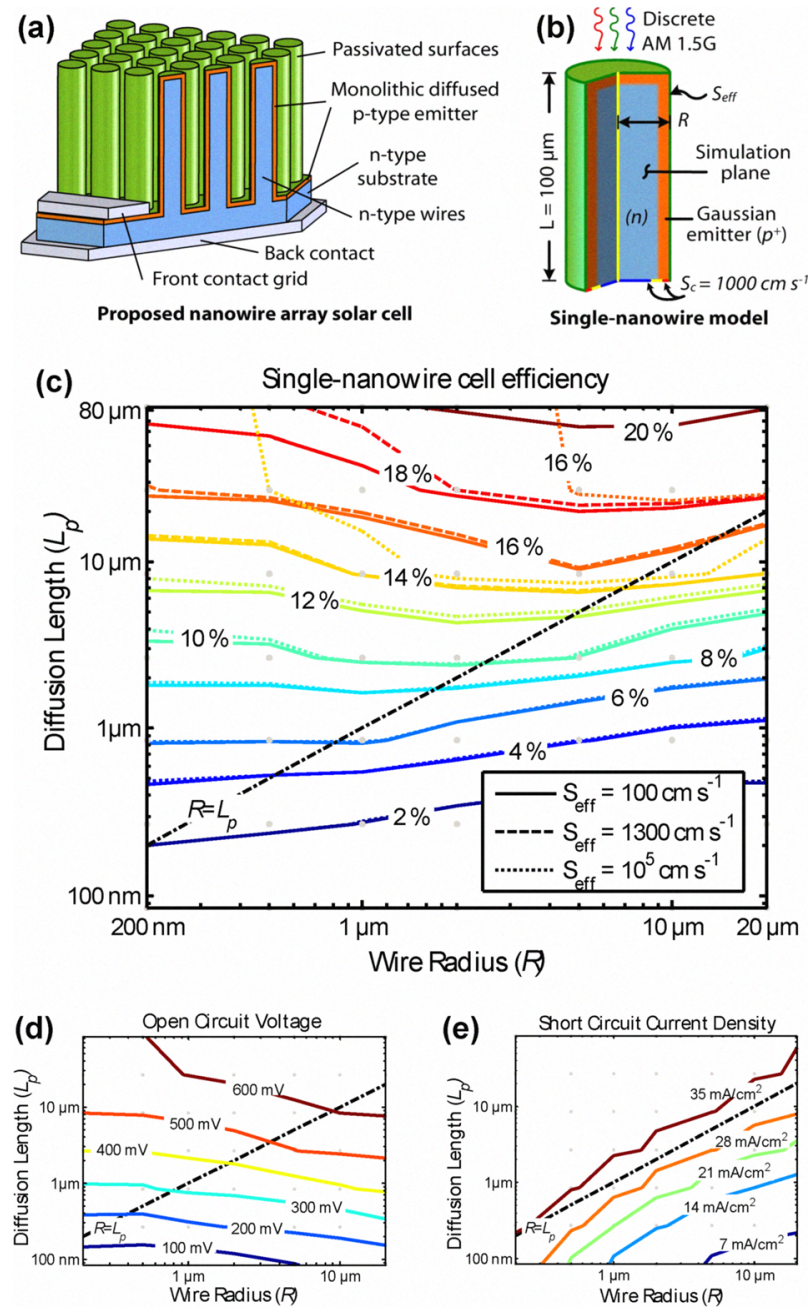

Fig. 3. Device physics model of single-wire solar cell. (a) Proposed wire array solar cell which serves as basis for single-wire model shown in (b), where the geometry and boundary conditions of the model are illustrated. (c) Simulated efficiency of single-nanowire solar cell model as a function of wire radius and diffusion length, calculated at three values of surface recombination velocity. The light gray circles represent actual devices which were simulated to cover this parameter space. (d) and (e) show simulated $V_{O C}$ and $J_{S C}$, respectively, of the nanowire device for $S_{\text {eff }}=1300 \mathrm{~cm} / \mathrm{s}$. The cell thickness limits $J_{S C}$ to $37 \mathrm{~mA} / \mathrm{cm}^{2}$.

in 2-dimensional cylindrical coordinates, which properly models a $3 D$ radial junction wire device under vertical illumination.

The single-wire model in figure $3(\mathrm{~b})$ was chosen to represent, with minimal complexity, the wire array cell that might reasonably be fabricated shown in figure 4(a). An n-type wire core was chosen with doping of $10^{18} \mathrm{~cm}^{-3}$, since our reactor can produce wires of this doping type and density. A Gaussian boron profile was chosen for the emitter, with surface concentration of $5 \times 10^{18} \mathrm{~cm}^{-3}$ and junction depth of $50 \mathrm{~nm}$, representing the approximate profile which could result from a boron diffusion step on the as-grown wire array. The wire length of $100 \mu \mathrm{m}$ was selected as a compromise between the optical thickness required for maximal absorption, and the increased computational resources required to model thicker cells. This thickness absorbs $\sim 90 \%$ of incident solar power [1] and limits maximally achievable current density to approximately $37 \mathrm{~mA} \mathrm{~cm}^{-2}$. A $5 \mathrm{~nm}$ oxide was placed at the wire side and top surfaces to allow surface recombination to be modeled at these surfaces. Ideal ohmic contacts were used at the base of the wire device. To approximate the array cell, where the growth substrate and monolithic emitter form the contacts to the base and emitter, respectively, of each wire, a minority carrier surface recombination velocity of $1000 \mathrm{~cm} \mathrm{~s}^{-1}$ was assigned to each of the contacts to the single-wire cell model.

The Sentaraus Device program calculates the terminal current of the solar cell model by simultaneously solving the electron and hole continuity equations and the Poisson equation within the device [15]. Unless otherwise specified, the default parameters for Si were used for all physical processes. A drift-diffusion model for carrier transport was employed, with doping-dependent mobility. Shockly-Reed-Hall (SRH) and Auger recombination were considered. For the purpose of generality, the SRH recombination was modeled throughout the wire as a single trap located at mid-gap, rather than considering the trapping dynamics introduced by catalyst metal incorporation. The lifetime was varied to obtain the desired diffusion length within the base. Depending on the actual recombination mechanisms and impurity distributions within the wires, this approach may underestimate depletion region recombination. Solar illumination was modeled by a 20 point discretization of the above-bandgap AM $1.5 \mathrm{G}$ spectrum, which preserved the incident power density, photon density, and overall shape of the relevant AM $1.5 \mathrm{G}$ spectrum. Dark and light $\mathrm{J}-\mathrm{V}$, spectral response, and SPCM/LBIC measurements were simulated for $\mathrm{Si}$ wire cells of varying radius $(R=0.2-20 \mu \mathrm{m})$, diffusion length $\left(L_{p} \approx 80 \mathrm{~nm}-80 \mu \mathrm{m}\right)$, and surface recombination velocity $\left(S_{\text {eff }}=100,1300,10^{5} \mathrm{~cm} \mathrm{~s}^{-1}\right.$.)

Figure 3(c) shows the simulated efficiency contours of the single-wire solar cell, indicating that the cell is capable of conversion efficiencies that exceed $20 \%$ within the considered parameter space. Figures 4 (d) and (e) show the $V_{\mathrm{OC}}$ and Isc contours, respectively. As predicted, the cell is capable of obtaining optimal Isc at arbitrarily low diffusion lengths, as long as the wire radius is less than the diffusion length. However, the $V_{O C}$ decreases rapidly at lower diffusion lengths, more so for smaller wire radius. Thus, for any given diffusion length, optimal cell efficiency occurs when $R \approx 1 / 2 L_{p}$. The cell is remarkably insensitive to surface recombination on the wire top and sidewalls, since the bulk absorber (core) is shielded from all surfaces by the active emitter layer. Only in cases of extreme $\left(S_{\text {eff }}=10^{5} \mathrm{~cm} \mathrm{~s}^{-1}\right)$ recombination is performance effected, in this case due mostly to the lowered blue response due to an effectively inactive emitter at the top of the wire. 
To obtain a refined estimate of achievable cell efficiencies based on experimentally measured wire properties, we consider the case of $L_{p}=3.0 \mu \mathrm{m}$, corresponding to a typical value observed in Au-catalyzed wires, and $L_{p}=$ $10 \mu \mathrm{m}$, a value consistent with experimental results for $\mathrm{Ni}-$ catalyzed wires. In both cases, the experimentallyinferred upper limit on surface recombination velocity is also applied. The radius of each device was chosen to be near the optimal radius predicted by figure $3(\mathrm{c}): 1.5 \mu \mathrm{m}$ for the $L_{p}=3.0 \mu \mathrm{m}$ case and $5 \mu \mathrm{m}$ for the $L_{p}=10 \mu \mathrm{m}$ case. These simulated cells exhibit power conversion efficiencies of $11 \%$ and $17 \%$, respectively. As a point of comparison, we simulated planar-junction cells with identical doping profiles and carrier lifetimes, with contact surface recombination velocities of $1000 \mathrm{~cm} \mathrm{~s}^{-1}$ to approximate the effects of passivation and a back-surface field in a planar cell. The simulated $\mathrm{J}-\mathrm{V}$ performance of these cells is shown in figure 4. As expected, the radial junction cells exhibit much higher current density, at the cost of cell operating voltage. Increasing the thickness of a single-wire cell to $300 \mu \mathrm{m}$ yields predicted efficiencies of over $18 \%$ for $L_{p}=10 \mu \mathrm{m}$.

It should be noted that this single-wire solar cell model does not account for many important complications associated with a cell based on arrays of many wires, namely the optical properties of such arrays. There are significant challenges in the way of efficient absorption within large wire arrays, including geometrical and practical limits on wire packing density. However, disordered films of sub-wavelength nanowires have been reported with reasonable optical absorption for photovoltaic applications [16-18]. For larger-diameter wires and ordered arrays, there are other potential optical benefits, since each vertical wire can function as a waveguide, and the array can function as a photonic bandgap crystal. A detailed analysis of this topic is necessary to supplement the device physics modeling presented here.

\section{CONCLUSIONS}

Solar cells based on arrays of Si wires are a promising approach to reducing the cost of solar cell production. We have measured diffusion lengths within Si wires which exceed $10 \mu \mathrm{m}$, and simulated an $17 \%$ efficient singlenanowire solar cell device with this diffusion length. It is foreseeable that diffusion lengths could be improved through growth condition alterations, surface passivation, or impurity gettering -- which has the potential to further improve the potential efficiency of this solar cell design.

\section{ACKNOWLEDGEMENTS}

The authors would like to thank Melissa Archer, Ryan Briggs, Bruce Brunschwig, Carol M. Garland, Ali Ghaffari and the micro/nano lab, David Knapp, Jim Maiolo, Stephen Maldonado, Evan and April Neidholdt, Domenico Pacifici, Katherine Plass, Jessica Roberts, Josh Spurgeon, and Luke Sweatlock.

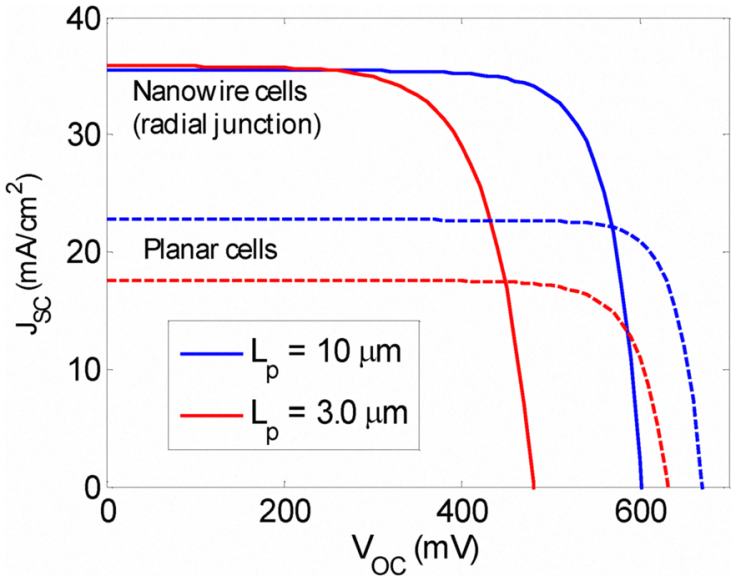

Fig. 4. Simulated AM 1.5G J-V performance of singlenanowire radial junction cells compared to planar cells with identical junction profiles and diffusion lengths. The nanowire cell efficiencies are $17 \%$ and $12 \%$, compared respectively to $13 \%$ and $8.8 \%$ for planar cells.

This work was supported by BP, the Department of Energy, Office of Basic Energy Sciences, and the Center for Science and Engineering of Materials, an NSF Materials Research Science and Engineering Center at Caltech.

\section{REFERENCES}

[1] B. M. Kayes, H. A. Atwater, and N. S. Lewis, "Comparison of the Device Physics Principles of Planar and Radial P-N Junction Nanorod Solar Cells", J. Appl. Phys., 97, pp. 114302-11, 2005.

[2] R. M. Swanson, "Approaching the 29\% Limit Efficiency of Silicon Solar Cells," in Photovoltaic Specialists Conference, 2005. Conference Record of the Thirty-first IEEE, 2005, pp. 889-894.

[3] R. S. Wagner and W. C. Ellis, "Vapor-LiquidSolid Mechanism of Single Crystal Growth", Appl. Phys. Lett., 4, pp. 89-90, 1964.

[4] J. M. Spurgeon, K. E. Plass, B. M. Kayes, H. A. Atwater, and N. S. Lewis, "Repeated Epitaxial Growth and Transfer of Arrays of Patterned, Vertically Aligned, Crystalline Si Wires from a Single Si(111) Substrate", In preparation.

[5] W. P. Rahilly, "Vertical Multijunction Solar Cells," in Record of the Ninth IEEE Photovoltaic Specialists Conference, Silver Spring, MD, USA, 1972, pp. 44-52.

[6] J. E. Allen, E. R. Hemesath, D. E. Perea, J. L. Lensch-Falk, Liz.Y, F. Yin, M. H. Gass, P. Wang, A. L. Bleloch, R. E. Palmer, and L. J. Lauhon, "High-Resolution 
Detection of Au Catalyst Atoms in Si Nanowires", Nat Nano, 3, pp. 168-173, 2008.

[7] M. D. Kelzenberg, D. B. Turner-Evans, B. M. Kayes, M. A. Filler, M. C. Putnam, N. S. Lewis, and H. A. Atwater, "Photovoltaic Measurements in Single-Nanowire Silicon Solar Cells", Nano Lett., 8, pp. 710-714, 2008.

[8] B. Tian, X. Zheng, T. J. Kempa, Y. Fang, N. Yu, G. Yu, J. Huang, and C. M. Lieber, "Coaxial Silicon Nanowires as Solar Cells and Nanoelectronic Power Sources", Nature, 449, pp. 885-889, 2007.

[9] B. M. Kayes, M. A. Filler, M. C. Putnam, M. D. Kelzenberg, N. S. Lewis, and H. A. Atwater, "Growth of Vertically Aligned Si Wire Arrays over Large Areas ( $>1$ Cm[Sup 2]) with Au and Cu Catalysts", Appl. Phys. Lett., 91, pp. 103110-3, 2007.

[10] W. M. Bullis, "Properties of Gold in Silicon", Solid-State Electron., 9, pp. 143-168, Feb. 1966.

[11] J. Crank, in The Mathematics of Diffusion, 2 ed: Oxford University Press, 1975, p. 69.

[12] A. A. Istratov and E. R. Weber, "Electrical Properties and Recombination Activity of Copper, Nickel and Cobalt in Silicon", Appl. Phys. A: Mater. Sci. Process., 66, pp. 123-136, 1998.
[13] A. L. Fahrenbruck and R. H. Bube, "Fundamentals of Solar Cells: Photovoltaic Solar Energy Conversion," New York: Academic Press, 1983, p. 83.

[14] S. M. Sze and K. N. Kwok, Physics of Semiconductor Devices. Hoboken, NJ: John Wiley \& Sons, 2007.

[15] Synopsys_Inc., "Sentaurus Device User Guide," Version A-2007.12 ed, 2007.

[16] L. Tsakalakos, J. Balch, J. Fronheiser, B. A. Korevaar, O. Sulima, and J. Rand, "Silicon Nanowire Solar Cells", Appl. Phys. Lett., 91, pp. 233117-3, 2007.

[17] K. Peng, Y. Xu, Y. Wu, Y. Yan, S.-T. Lee, and J. Zhu, "Aligned Single-Crystalline Si Nanowire Arrays for Photovoltaic Applications", Small, 1, pp. 1062-1067, 2005.

[18] L. Hu and G. Chen, "Analysis of Optical Absorption in Silicon Nanowire Arrays for Photovoltaic Applications", Nano Lett., 7, pp. 3249-3252, 2007. 